\title{
(Aminophosphane)gold(I) and silver(I) complexes as antibacterial agents
}

\section{Lourdes Ortego ${ }^{a}$, Jesús Gonzalo-Asensio ${ }^{b, c}$ Antonio Laguna ${ }^{a}$, M. Dolores Villacampa $^{\mathrm{a}}{ }^{*}$ M. Concepción Gimeno ${ }^{\mathrm{a}}{ }^{* 1}$}

a Departamento de Química Inorgánica, Instituto de Síntesis Química y Catálisis Homogénea (ISQCH), Universidad de Zaragoza-CSIC, E-50009 Zaragoza, Spain

b Grupo de Genética de Micobacterias, Departamento de Microbiología, Medicina Preventiva y Salud Pública, Facultad de Medicina, Universidad de Zaragoza, E-50009 Zaragoza, Spain.

c CIBER de Enfermedades Respiratorias, Instituto de Salud Carlos III, E-28029 Madrid, Spain.

\section{ABSTRACT}

This manuscript describes the synthesis of new $\mathrm{Au}(\mathrm{I})$ and $\operatorname{Ag}(\mathrm{I})$ complexes with aminophosphane ligands and a study of their antibacterial activity against Gramnegative Salmonella enterica serovar typhimurium and Escherichia coli and Grampositive Listeria monocytogenes and Staphylococcus aureus. The bactericidal assays revealed the effectiveness of these compounds on paradigm Gram-negative and Grampositive pathogens, showing a moderate antimicrobial activity, comparable with the antibiotics of reference, for all gold(I) complexes and the silver(I) complexes without coordinated $\mathrm{PPh}_{3}$ groups. For those complexes that were found to show inhibitory

\footnotetext{
1 Corresponding authors: M. D. Villacampa: dvilla@unizar.es, M. C. Gimeno: gimeno@unizar.es Tel.: +34976762291, fax.: +34976761187
} 
activity, serial dilutions in liquid broth method were performed for determination of MIC (minimum inhibitory concentration) and MBC (minimum bactericidal concentration).

Keywords: gold(I); silver(I); aminophosphine ligands; Antibacterial activity; Gramnegative; Gram-positive

\section{Introduction}

The use in medicine of penicillin in the 1940s, erythromycin in the 1950s and methicillin in the 1960s greatly contributed to control bacterial diseases. However, the extended and uncontrolled use of antibiotics soon brought the need for the development of new antimicrobial agents because of the rapid increase of bacterial resistance, decreasing antibiotic efficacy and complicating the treatment of infections. The acquired bacterial resistance to vancomycin was slower and required nearly thirty years to found antibiotic resistant strains [1]. Since then, a large number of transition metal compounds have been screened against bacteria and fungi. For gold(I), research has been principally focused on compounds with a SAuP or NAuP core, exploiting the analogy to Auranofin [2]. Studies have shown good bactericidal or bacteriostatic results [3-5], some of them including Auranofin and related complexes have shown as potent inhibitors of methicillin-resistant Staphylococcus aureus [6], and they also present antitumor, antimalarial, or antirheumatic activities [7]. Other important type of metal complexes presenting good antibacterial activity are those related to N-heterocyclic carbene ligands NHCs, especially silver but also gold complexes [8].

Silver(I) compounds also show a wide spectrum of effective antimicrobial activity. Silver nitrate has been used since the seventeenth century for the prevention of neonatal conjunctivitis and is still in use today [9]. The most commonly used silver compound is silver(I) sulfadiazine ([Ag((4-aminophenyl)sulfonyl)(pyrimidin-2-yl)azanide $]_{\mathrm{n}}$, which is widely used in medicine to treat and prevent the development of bacterial infections in skin burns [10]. Nowadays numerous silver(I) compounds with AgN, AgP or NAgP unit have been synthesized [11-14], and more recently $\mathrm{AgC}$ from NHCs $[15,16]$ and have presented cytotoxic activity against Gram-positive and Gram-negative bacteria, 
although the mechanisms of action of silver(I) compounds are not completely understood.

Infections caused by Gram-positive and Gram- negative bacteria are of major concern due to the increased incidence of drug resistant strains. The food-borne Salmonella typhimurium and Escherichia coli are the most prevalent Gram-negative pathogens in humans and animals. These bacteria cause enteric diseases that are commonly treated by $\beta$-Lactam antibiotics. However, the prolonged administration of these antibiotics have resulted in a striking correlation between drug use and the emergence of antibiotic resistant strains. $\beta$-Lactam antibiotics interfere with bacterial cell wall biosynthesis that eventually leads to cell death. The mechanism of action is to bind covalently to their targets, the penicillin-binding proteins (PBPs), enzymes involved in cell wall synthesis in the cytoplasmic membrane of the bacteria. Antibiotics penetrate the outer membrane of gram-negative bacteria through protein pores called porins. The acquired resistance to $\beta$-lactam antibiotics may be due to a range of diverse factors: mutations in the PBPs that reduce or prevent the binding to the antibiotic, porin mutations which preclude or reduce outer-membrane permeability [17], hyper-expression of efflux pumps that expel antibiotics outside the bacteria or $\beta$-lactamases produced by bacteria to cleave the antibiotic. The combination of these factors results in Gram-negative resistance to $\beta$ Lactam antibiotics $[18,19]$.

With regard to Gram-positive organisms, Listeria monocytogenesis is a food-borne human pathogen responsible for listeriosis, a disease that although not common in healthy people, can cause severe diseases including abortions and meningitis. The number of listeriosis cases has increased in recent years in several industrialized countries [20-23] and although treatment with ampicillin or penicillin in combination with gentamicin (or other aminoglycoside) [24] is effective, the recent increase in antibiotic resistance is a concern. For example, strains N53-1 and 4446 are resistant to gentamicin and other aminoglycosides, and some strains are able to persist within different types of food processing plants for years and consequently are repeatedly exposed to biocides, something that could potentially impact on bacterial resistance [2528].

Staphylococcus aureus is another Gram-positive bacteria causative of serious hospitalacquired infections. Nowadays strains with intermediate levels of resistance to 
vancomycin or even resistant appear more frequently [29]. One reason for this could be the bifunctional acetyltransferase(6')-Ie-phosphotransferase(2")-Ia[AAC(6')-IeAPH(2")-Ia], the most important aminoglycoside-resistance enzyme in Gram-positive bacteria, that confers resistance to almost all known aminoglycoside antibiotics in clinical use [30]. However resistance is not only related to aminoglycoside antibiotics like vancomycin, there are $S$. aureus strains resistant to methicillin that are also resistant to practically all $\beta$-Lactam group (penicillin, oxacillin, ampicillin, amoxicillin and augmentin), and macrolides (clindamycin and azithromycin). These multidrug-resistant strains are spreading outside hospitals and represent a serious concern [31] and sensitive to the aminoglycoside group (amikacin), macrolide antibiotics (nitrofurantoin) and the quinolone group (ciprofloxacin, ofloxacin and norfloxacin) [32-35].

In this work we describe the synthesis and structural characterization of new gold and silver complexes with aminophosphane ligands. These ligands were chosen because other phosphane-gold derivatives are known to have antibacterial activity and because the presence of the amine group allows and easy functionalisaton, making possible the introduction of relevant biological moieties. These compounds were tested in vitro to study their activity against Gram-negative $S$. typhimurium SV5015 and E. coli ATCC 10536 strains and the Gram-positive L. monocytogenes EGD-e and S. aureus ATCC 11632 strains using the paper disc diffusion method (for the qualitative determination) and for those complexes that were found to show inhibitory activity, serial dilutions in liquid broth method were performed (for determination of MIC (minimum inhibitory concentration) and $\mathrm{MBC}$ (minimum bactericidal concentration)). This paper is a continuation of a previous study using the same ligand [36], in which the susceptibility of (aminophosphane)gold(I) thiolate complexes against Enterococcus faecalis ATCC 25923, S. aureus ATCC 29213 and E. coli TG1 was demonstrated. Some of them were found to exhibit powerful antibacterial activity, being more efficient against Grampositive microorganisms. 


\section{Results and discussion}

\subsection{Synthesis and Characterization}

Herein we report the synthesis and characterization of a series of gold(I) and silver(I) derivatives with two different aminophosphane ligands: 2(diphenylphosphanylamino)pyridine $\quad\left(\mathrm{Ph}_{2} \mathrm{PNHpy}\right) \quad$ (Scheme 1$)$ and 3(diphenylphosphanylamino)-1,2,4-triazole $\quad\left[\mathrm{Ph}_{2} \mathrm{PNH}(\mathrm{Htrz})\right] \quad($ Scheme 2) [37]. Spectroscopic characterizations of the metal complexes were performed using IR, ESI+ mass spectra, and multinuclear NMR spectroscopy, ${ }^{1} \mathrm{H},{ }^{19} \mathrm{~F}\left\{{ }^{1} \mathrm{H}\right\},{ }^{31} \mathrm{P}\left\{{ }^{1} \mathrm{H}\right\}$, and ${ }^{13} \mathrm{C}\left\{{ }^{1} \mathrm{H}\right\}$ NMR.

\section{Scheme 1}

\section{Scheme 2}

In the ESI+ (positive mode of electrospray ionization) mass spectra of 3-6, 8, 9, 12 and $\mathbf{1 4}$ the cation peaks [M-OTf] $]^{+}$are observed and in $\mathbf{1 0}$ the protonated molecular peaks [M $+\mathrm{H}]^{+}$are seen. The IR spectra of $\mathbf{2}$ and $\mathbf{1 1}$ show the bands corresponding to the presence of the pentafluorophenyl groups bonded to the gold atom. Their ${ }^{19} \mathrm{~F}\left\{{ }^{1} \mathrm{H}\right\} \mathrm{NMR}$ spectra show three signals corresponding to ortho, meta and para fluorine atoms, respectively, of the pentafluorophenyl ring with small shifts compared to the starting material $\left[\mathrm{Au}\left(\mathrm{C}_{6} \mathrm{~F}_{5}\right)(\right.$ tht $\left.)\right]$ (tht $=$ tetrahydrothiophene). The ${ }^{1} \mathrm{H}$ NMR spectra of the compounds in d6-DMSO or d6-acetone present the expected resonances for all of the protons (see experimental section) and show a low or high-field shift of the aromatic protons indicatives of the successful coordination of the metal to the ligand through the phosphorus $\left(\mathbf{1 - 5 , 1 0 - 1 2 )}\right.$ or the phosphorus and the nitrogen atom $\left(\mathbf{6 - 9 , 1 3 - 1 4 )}\right.$. The ${ }^{1} \mathrm{H}$ NMR spectra of complexes 3, 4, 8 and $\mathbf{9}$ show the corresponding phosphine group signal, indicative of the coordination of one or two phosphine group. The ${ }^{31} \mathrm{P}\left\{{ }^{1} \mathrm{H}\right\} \mathrm{NMR}$ spectra provide a great deal of information about the present series of complexes. The gold compounds show single resonances 54.0 (1), 65.3 (2), 64.8 (3), 64.8 (4), 62.3 (5), 54.0 (10), 68.9 (11), 68.7 (12) significantly downfield relative to their precursors, 25.5 (L1) or 36.9 (L2). The silver ${ }^{31} \mathrm{P}\left\{{ }^{1} \mathrm{H}\right\}$ NMR spectra at room temperature display one broad signal for complexes $\mathbf{6}, 7,13$ and 14 or two broad resonances for complexes 8 
and 9. At $203 \mathrm{~K}$ the ${ }^{31} \mathrm{P}\left\{{ }^{1} \mathrm{H}\right\}$ NMR spectra of complexes 6, 7 and $\mathbf{1 4}$ show two doublets due to coupling with ${ }^{107} \mathrm{Ag}$ and ${ }^{109} \mathrm{Ag}$ nuclei. The ${ }^{31} \mathrm{P}\left\{{ }^{1} \mathrm{H}\right\}$ NMR spectrum at $203 \mathrm{~K}$ of complex 9 shows signals that correspond to an $\mathrm{AB}_{2}$ system: two doublets centered at 9.9 and $9.3 \mathrm{ppm}$ and two multiplets centered at 23.9 and $21.8 \mathrm{ppm}$. At $203 \mathrm{~K}$ complex 8 shows a complicated spectrum with two groups of signals: one that appears to correspond to an $\mathrm{AB}_{2}$ system, with equal shifts to complex 9 and other that appears to correspond to complex 7. With the information obtained from ${ }^{31} \mathrm{P}\left\{{ }^{1} \mathrm{H}\right\}$ NMR studies at low temperature we propose the existence in solution of complex 8 as an equilibrium between two species, one without a $\mathrm{PPh}_{3}$ group (7) and the other with two $\mathrm{PPh}_{3}$ groups (9) (Figure 1) [14, 38-42].

Fig. 1

The reaction between $\mathbf{L} 2$ and $\left[\mathrm{Au}(\mathrm{tht})_{2}\right](\mathrm{OTf})$ in a $2: 1$ ratio or $\left[\mathrm{Au}(\mathrm{OTf})\left(\mathrm{PR}_{3}\right)\right]\left(\mathrm{R}_{3}=\right.$ $\mathrm{PPh}_{3}, \mathrm{PPh}_{2}$ py) in a 1:1 ratio gave complex (12). In the ${ }^{1} \mathrm{H}$ NMR spectra we find the expected displaced resonances for the ligand $\mathbf{L} 2$ protons but the corresponding phosphine group signal in the reaction with $\left[\mathrm{Au}(\mathrm{OTf})\left(\mathrm{PR}_{3}\right)\right]\left(\mathrm{R}_{3}=\mathrm{PPh}_{3}, \mathrm{PPh}_{2} \mathrm{py}\right)$ do not appear. The ${ }^{31} \mathrm{P}\left\{{ }^{1} \mathrm{H}\right\}$ NMR spectra show a signal significantly downfield relative to the precursor $\mathbf{L} 2$.

\section{2. Crystal structure description}

We confirmed the molecular structures of complexes 6, 9 and $\mathbf{1 0}$ by an X-ray crystallographic study, although the last one was previously published [36]. Complex 6 (Figure 2) crystallizes in a monoclinic system with two independent units in the asymmetric unit. There are no important geometric differences between the two independent molecules, which shown similar Ag-N and Ag-P bond distances and these are also comparable to those found in other derivatives with the same N-Ag-P core where the silver atom is in tetracoordinated around. Both silver atoms are in a highly distorted tetrahedral geometry caused by the aminophosphane ligand that forces chelation angles from $75.74(6)^{\mathrm{o}}$ to $77.29(6)^{\mathrm{o}}$.

There are several hydrogen bond in the molecule involving the oxygen of the triflate anions as an acceptor and the protons of the amide group (NHpy) of the ligand as a donor of protons; in all the cases the angles $\mathrm{D}-\mathrm{H} \cdots \mathrm{A}$ are close to $180^{\circ}$. 
Fig. 2

The structure of complex 9 has also been established by X-ray diffraction and the molecule can be seen in Fig. 3. The geometry around the silver center is tetrahedral with the main distorsion arising from the bite angle of the phosphinoamine, 74.99(12) ${ }^{\mathrm{o}}$. The Ag-P and Ag-N distances are very similar to those found in complex 6.

Fig. 3

\subsection{Antibacterial activity}

All the complexes synthesized were tested for their in vitro antibacterial activity against Gram-negative bacteria S. typhimurium SV5015 and E. coli ATCC 10536 and Grampositive L. monocytogenes EGD-e and S. aureus ATCC 11632. Ampicillin, kanamycin, gentamicin, chloramphenicol, vancomycin, erythromycin and rifampicin were used as standard drugs for Gram-positive and/or Gram-positive bacteria. Structures of these drugs are shown in Figure 4.

Fig. 4

The qualitative determination for the complexes studied is presented in Tables 1 and 2. Our previous report shows that the ligands have no bactericidal activity, whereas some of the metal complexes show activity under identical experimental conditions.

All the compounds were insoluble in water and the assay was carried out using DMSO as solvent. The solvent DMSO (in the same percentage used to dissolve the compounds) was also used as a control to confirm that it did not inhibit bacterial growth. The results indicate that the metal was responsible for the observed activities.

\section{Table 1}

\section{Table 2}

In the qualitative determination it was not possible to screen the activity of complexes 2 , 5, and 12 due to solubility problems. None of the silver(I) and gold(I) compounds studied were effective against Gram-negative bacteria (Table 1), however the results were different for Gram-positive bacteria (Table 2) with the complexes showing biological activity. Figure 5 shows zones of bacterial growth inhibition for some of the complexes by the disc diffusion method. 
The different antibacterial behavior of complexes might be related to the cell wall, since this is the first structural difference between Gram-positive and Gram-negative bacteria that represents the primary target for antimicrobial agents. Gram-positive bacteria possess a thick cell wall of peptidoglycan whereas Gram-negative bacteria have a thin cell wall of peptidoglycan surrounded by a second lipid membrane containing lipopolysaccharides and lipoproteins. Hence, it is tempting to speculate that the different bactericidal behavior of compounds can be explained by their ability to pass through the membrane and somehow interact; producing changes that eventually cause the death of the bacteria $[43,44]$. The interaction is based on the strong attraction of positively charged compounds. If compounds can form bond complexes or interact electrostatically with the cell wall, it is very probable that the compound will possess biocide activity. This phenomenon occurs more readily with Gram-positive bacteria because the pores of peptidoglycan cell wall are not efficient barriers for the compounds of determined size [45]. Furthermore, it has been shown that some pyrazine functionalized $\mathrm{Ag}(\mathrm{I})$ and $\mathrm{Au}(\mathrm{I})$-NHC complexes strongly binds both Lys and Dap-type peptidoglycan layers, which may be the reason for damage to the bacterial cell wall. [46].

The negative bactericidal results of silver(I) complexes are in agreement with previous studies that explain that $\mathrm{Ag}(\mathrm{I})-\mathrm{P}$ complexes show no activity, very likely because there is no exchange with biomolecules. The antimicrobial properties of silver(I) complexes depend upon the fast rate of ligand exchange of the metal ion in the biological system (more than solubility, charge, chirality or degree of polymerization) and this correlates to the nature of the donor atoms coordinated to the silver [47-51]. The silver(I) compounds with weak $\mathrm{Ag}-\mathrm{O}$ and $\mathrm{Ag}-\mathrm{N}$ bonds exhibit a wider spectrum of antibacterial activity because they can more readily exchange their N- or O- ligands for O-, N-, Sdonor atoms of amino acids or nucleotides [52]. Ag(I)-S complexes show a narrower spectrum of activity compared to Ag-N and Ag-O [53-55] and silver(I)-P complexes [56] have shown no activity against bacterial strains, yeast and molds [57]. Gold(I) results also are in agreement with results found in the literature. The complexes with a $\mathrm{S}-\mathrm{Au}-\mathrm{P}$ bond show better bactericidal properties against Gram-positive bacteria than 
those with an N-Au-P core [7,36] (Table 1 and 2). Gold(I) phosphine derivatives present no activity against $E$. coli and $S$. typhimurium (Table 1 and 2), although in some water soluble gold(I) phosphine complexes have been reported to have activity in these bacteria [58].

For the tested Gram-positive bacteria, results are positive. For L. monocytogenes, gold(I) compounds show bacterial activity and some of them present a bigger inhibitory zone compared with control antibiotics. Silver(I) compounds with L2 (13 and 14) present biological properties. Assays with $S$. aureus shows practically identical results to L. monocytogenes. Only one silver(I) compound (14) presents inhibitory activity and all gold(I) compounds show diameters of zones of inhibition larger than that of vancomycin, the only antibiotic used as reference that presented bactericidal activity in the strain. For both strains silver compounds without phosphine $\left(\mathrm{PPh}_{3}\right)$ groups coordinated are the complexes showing antibacterial activity. Also, in the chemical discussion we present the existence in solution of equilibrium between 8 and compounds 9 and 7. If there is equilibrium, a similar bactericidal behavior of both complexes as we found in the study with Gram-positive bacteria is expected.

It was determined the minimum inhibitory concentrations (MICs) and minimal bactericidal concentrations (MBCs) for complexes displaying zone inhibition in the disc diffusion method (Table 3 ). The results show that the majority of studied complexes displayed bacteriostatic activity as assessed by the lower MIC compared to the MBC values. In some cases where the MBC could be determined, the values were the same as MIC, indicating a potential bactericidal activity for these compounds.

Table 3

\section{Conclusions}

A series of new silver(I) and gold(I) derivatives have been synthesized and characterized as a continuation of a previous research of gold(I) thiolate complexes using the same aminophosphane ligands. The bactericidal assays revealed the effectiveness of all the tested compounds on Gram-negative and in Gram-positive bacteria, showing a moderate antimicrobial activity for all gold(I) complexes and the 
silver(I) derivatives without $\mathrm{PPh}_{3}$ groups coordinated, which is comparable to the reference antibiotics. These results confirm the influence of the atoms coordinated to the metal center found in the literature, and suggest that the mechanism for the antibacterial action might be related to the inhibition of peptidoglycan synthesis in the cell wall because of the specific selectivity to Gram-positive versus Gram-negative bacteria. This finding contributes to fill the pipeline of antimicrobial compounds that could be potentially useful to treat emerging drug resistant pathogens. 


\section{Experimental section.}

\subsection{Chemistry}

Instrumentation: IR spectra were recorded in the range 4000-200 cm-1 with a PerkinElmer Spectrum 100 spectrophotometer on solid samples by using an ATR accessory. C, $\mathrm{H}$ and $\mathrm{N}$ analyses were carried out with a Perkin-Elmer 2400 Series 2 microanalyser. Mass spectra were recorded with a VG Austopec by using the ESI technique. NMR spectra were recorded in $\left[\mathrm{D}_{6}\right] D M S O, C D C l_{3}$ or $\left(C_{3}\right)_{2} C O$ solution, most of them with a Bruker ARX 400 spectrometer; some of the ${ }^{13} \mathrm{C}\left\{{ }^{1} \mathrm{H}\right\}$ NMR spectra were recorded with a Bruker ARX 300 spectrometer. Chemical shifts are cited relative to Syme $4\left({ }^{1} \mathrm{H},{ }^{13} \mathrm{C}\right.$, external) and $85 \% \mathrm{H}_{3} \mathrm{PO}_{4}\left({ }^{31} \mathrm{P}\right.$, external). Unfortunately, in the ${ }^{13} \mathrm{C}\left\{{ }^{1} \mathrm{H}\right\} \mathrm{NMR}$ spectra of several complexes we did not observe all of the signals expected, probably as a consequence of the poor solubility of the complexes.

\subsubsection{Starting materials}

The phosphane ligands $\mathrm{Ph}_{2} \mathrm{PNHpy}(\mathbf{L 1})$ and $\left[\mathrm{Ph}_{2} \mathrm{PNH}(\mathrm{Htrz})\right]$ (L2) [37], the gold(I) complexes [AuCl$\left.\left(\mathrm{Ph}_{2} \mathrm{PNHpy}\right)\right]$ (2) and $\left[\mathrm{AuCl}\left\{\mathrm{Ph}_{2} \mathrm{PNH}(\mathrm{Htrz})\right\}\right]$ (10) [37] and the starting materials $\left.\left[\mathrm{Au}\left(\mathrm{C}_{6} \mathrm{~F}_{5}\right)(\mathrm{tht})\right][59],\left[\mathrm{Au}(\text { tht })_{2}\right](\mathrm{OTf})\right][60]$ and $\left[\mathrm{Ag}(\mathrm{OTf})\left(\mathrm{PPh}_{3}\right)\right][61]$ were prepared according to published procedures. $\left[\mathrm{Au}(\mathrm{OTf})\left(\mathrm{PPh}_{3}\right)\right]$ was obtained by reaction of $\left[\mathrm{AuCl}\left(\mathrm{PPh}_{3}\right)\right]$ [62] with $\mathrm{Ag}(\mathrm{OTf})$ in dichloromethane and used in situ. All other reagents were commercially available and used without further purification.

\subsubsection{Synthesis of metal complexes}

Synthesis of $\left[\mathrm{Au}\left(\mathrm{C}_{6} \mathrm{~F}_{5}\right)\left(\mathrm{PPh}_{2} \mathrm{NHpy}\right)\right](2)$

To a solution of L1 (0.055 g, $0.2 \mathrm{mmol})$ in $20 \mathrm{~mL}$ of dichloromethane was added $\left[\mathrm{Au}\left(\mathrm{C}_{6} \mathrm{~F}_{5}\right)(\mathrm{tht})\right](0.090 \mathrm{~g}, 0.2 \mathrm{mmol})$. The mixture was stirred for $1 \mathrm{~h}$ and then evaporation of the solvent to $c a .5 \mathrm{~mL}$ and addition of hexane gave complex 2 as a white solid. Yield: 0.093 g, $72.50 \%$. $\mathrm{C}_{23} \mathrm{H}_{15} \mathrm{~N}_{2} \mathrm{PF}_{10}$ (642.31): calcd. C, 42.92; H, 2.23; $\mathrm{N}, 4.48$, found $\mathrm{C}, 43.01 ; \mathrm{H}, 2.35 ; \mathrm{N}, 4.36$. IR $\left(\mathrm{cm}^{-1}\right): v(\mathrm{NH})=2978, v(\mathrm{C}=\mathrm{N})=1602$, $v(\mathrm{PN})=911, v\left(\mathrm{C}_{6} \mathrm{~F}_{5}\right) 1500,954,800 .{ }^{1} \mathrm{H} \mathrm{NMR}(400 \mathrm{MHz}, D M S O) \delta: 8.84(\mathrm{~d}, 1 \mathrm{H}, \mathrm{NH}$, 
$\left.{ }^{2} J_{\mathrm{PH}} 3.9 \mathrm{~Hz}\right), 8.07\left(\mathrm{~m}, 1 \mathrm{H}, H_{6}\right), 7.75\left(\mathrm{~m}, 4 \mathrm{H}, H_{P h}\right), 7.63\left(\mathrm{~m}, 7 \mathrm{H}, H_{P h y} 4\right), 7.03(\mathrm{~d}, 1 \mathrm{H}$, $\left.H_{3},{ }^{3} J_{\mathrm{HH}} 8.3 \mathrm{~Hz}\right), 6.88\left(\mathrm{~m}, 1 \mathrm{H}, H_{5}\right) .{ }^{31} \mathrm{P}\left\{{ }^{1} \mathrm{H}\right\}$ NMR (400 MHz, DMSO) $\delta: 65.3$ (s, 1P, $\left.P P h_{2}\right) .{ }^{19} \mathrm{~F}$ NMR (400 MHz, DMSO) $\delta:(-114.59)\left(\mathrm{m}, 2 \mathrm{~F}, F_{\text {ortho }}\right),(-158.43)\left(\mathrm{t}, 1 \mathrm{~F}, F_{\text {para }}\right.$, $\left.J_{\text {meta-para }} 21.2 \mathrm{~Hz}\right),(-161.74)\left(\mathrm{m}, 2 \mathrm{~F}, F_{\text {meta }}\right)$.

Synthesis of $\left[\mathrm{Au}\left(\mathrm{PR}_{3}\right)\left(\mathrm{PPh}_{2} \mathrm{NHpy}\right)\right](\mathrm{OTf})\left(\mathrm{R}_{3}=\mathrm{Ph}_{3}(3), \mathrm{Ph}_{2} \mathrm{Py}(4)\right)$.

To a solution of L1 $(0.055 \mathrm{~g}, 0.2 \mathrm{mmol})$ in $20 \mathrm{~mL}$ of dichloromethane was added $\left[\mathrm{Au}(\mathrm{OTf})\left(\mathrm{PPh}_{3}\right)\right](0.127 \mathrm{~g}, 0.21 \mathrm{mmol})$ or $\left[\mathrm{Au}(\mathrm{OTf})\left(\mathrm{PPh}_{2} \mathrm{py}\right)\right](0.128 \mathrm{~g}, 0.21 \mathrm{mmol})$. The mixture was stirred for $2 \mathrm{~h}$ and then evaporation of the solvent to $c a .5 \mathrm{~mL}$ and addition of hexane gave complexes $\mathbf{3}$ and $\mathbf{4}$ as white solids.

Complex 3. Yield: 0.147 g, $82.70 \% . \mathrm{C}_{36} \mathrm{H}_{30} \mathrm{AuF}_{3} \mathrm{~N}_{2} \mathrm{O}_{3} \mathrm{P}_{2} \mathrm{~S}$ (886.61): calcd. C, 48.77; $\mathrm{H}$, 3.41; N, 3.16; S, 3.62, found C, 49.32; H, 3.61, N, 3.29; S, 4.12. IR $\left(\mathrm{cm}^{-1}\right): v(\mathrm{NH})=$ $3224, v(\mathrm{C}=\mathrm{N})=1592, v(\mathrm{PN})=906, v_{\text {asym }}\left(\mathrm{SO}_{3}\right)=1273$ y $1248, v_{\text {sym }}\left(\mathrm{CF}_{3}\right)=1218$, $v_{\text {asym }}\left(\mathrm{CF}_{3}\right)=1150, v_{\text {sym }}\left(\mathrm{SO}_{3}\right)=1028 .{ }^{1} \mathrm{H}$ NMR $\left(400 \mathrm{MHz},\left(C D_{3}\right)_{2} C O\right) \delta: 7.78(\mathrm{~m}, 1 \mathrm{H}$, $\left.H_{6}\right), 7.51\left(\mathrm{~m}, 9 \mathrm{H}, H_{P h}{ }_{4}\right), 7.31\left(\mathrm{~m}, 11 \mathrm{H}, H_{P h}\right), 7.04\left(\mathrm{~d}, 1 \mathrm{H}, H_{3},{ }^{3} J_{\mathrm{HH}} 8.3 \mathrm{~Hz}\right), 6.81(\mathrm{~m}$, 1H, $\left.H_{5}\right) .{ }^{1} \mathrm{H}$ NMR (400 MHz, DMSO) $\delta: 8.99(\mathrm{~m}, 1 \mathrm{H}, N H), 7.71\left(\mathrm{~m}, 1 \mathrm{H}, H_{6}\right), 7.62(\mathrm{~m}$, $\left.1 \mathrm{H}, H_{4}\right), 7.45\left(\mathrm{~m}, 14 \mathrm{H}, H_{P h}\right), 7.18\left(\mathrm{~m}, 3 \mathrm{H}, H_{P h}\right), 6.93\left(\mathrm{~d}, 1 \mathrm{H}, H_{3},{ }^{3} J_{\mathrm{HH}} 8.2 \mathrm{~Hz}\right), 6.76(\mathrm{~m}$, $\left.1 \mathrm{H}, H_{5}\right) .{ }^{19} \mathrm{~F}$ NMR $\left(400 \mathrm{MHz},\left(C D_{3}\right){ }_{2} C O\right) \delta:-80.03$ (s, $\left.1 \mathrm{~F}, \mathrm{OTf}\right) .{ }^{31} \mathrm{P}\left\{{ }^{1} \mathrm{H}\right\} \mathrm{NMR}(400$ $\left.\mathrm{MHz},\left(C D_{3}\right)_{2} C O\right) \delta: 64.8\left(\mathrm{~s}, 1 \mathrm{P}, P P h_{2}\right), 37.0\left(\mathrm{~s}, 1 \mathrm{P}, P P h_{3}\right) .{ }^{13} \mathrm{C}\left\{{ }^{1} \mathrm{H}\right\} \mathrm{NMR}(400 \mathrm{MHz}$, DMSO) $\delta: 155.50\left(\mathrm{~s}, 1 \mathrm{C}, \mathrm{C}_{2}\right), 146.84\left(\mathrm{~s}, 1 \mathrm{C}, C_{6}\right), 138.25$ (s, $\left.1 \mathrm{C}, C_{4}\right), 133.69(1 \mathrm{C}$, $C_{\text {CipsoPh, }}$ d, $\left.{ }^{1} J_{\mathrm{CP}} 46.4 \mathrm{~Hz}\right), 133.08$ (d, $\left.1 \mathrm{C}, C_{\text {orthoPh }},{ }^{2} J_{\mathrm{CP}} 14.7 \mathrm{~Hz}\right), 131.09$ (d, $1 \mathrm{C}, C_{\text {orthoPh }}$,

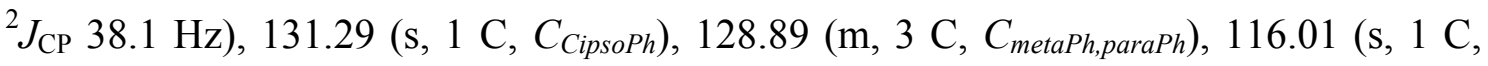
$\left.C_{5}\right), 112.15\left(\mathrm{~s}, 1 \mathrm{C}, C_{3}\right)$. MS (ESI+): $\mathrm{m} / \mathrm{z}(\%)=737.3\left([\mathrm{M}-\mathrm{OTf}]^{+}, 100\right), 752.2$ $\left(\left[\left(\mathrm{PPh}_{2} \mathrm{NHpy}\right)_{2} \mathrm{Au}\right]^{+}, 54.1\right)$.

Complex 4. Yield: 0.142 g, 80.31\%. $\mathrm{C}_{35} \mathrm{H}_{29} \mathrm{AuF}_{3} \mathrm{~N}_{3} \mathrm{O}_{3} \mathrm{P}_{2} \mathrm{~S}$ (887.60): calcd. C, 47.36; $\mathrm{H}$, $3.29 ; \mathrm{N}, 4.73$; S, 3.61, found C, 47.90; H, 3.61; N, 5.02; S, 3.62. IR $\left(\mathrm{cm}^{-1}\right): v(\mathrm{NH}) 3189$, $v(\mathrm{C}=\mathrm{N})=1590, v(\mathrm{PN})=910, v_{\text {asym }}\left(\mathrm{SO}_{3}\right)=1273$ y $1248, v_{\mathrm{sym}}\left(\mathrm{CF}_{3}\right)=1223, v_{\text {asym }}\left(\mathrm{CF}_{3}\right)=$ $\left.1150, v_{\mathrm{sym}}\left(\mathrm{SO}_{3}\right)=1028 .{ }^{1} \mathrm{H} \mathrm{NMR}\left(400 \mathrm{MHz},\left(C D_{3}\right)_{2} C O\right)\right) \delta: 8.79\left(\mathrm{~d}, 1 \mathrm{H}, H_{6},{ }^{3} J_{H H} 4.5\right.$ $\mathrm{Hz}), 8.27$ (s br, 1H, NH), $7.88\left(\mathrm{~m}, 6 \mathrm{H}, H_{P h y}\right.$ ), 7.62 (m, 20H, $\left.H_{P h}, 4,3^{\prime}, 4^{\prime}, 5^{\prime}\right), 7.14(\mathrm{~d}, 1 \mathrm{H}$, $\left.\left.H_{3},{ }^{3} J_{H H} 8.3 \mathrm{~Hz}\right), 6.90\left(\mathrm{~m}, 1 \mathrm{H}, H_{5}\right) .{ }^{31} \mathrm{P}\left\{{ }^{1} \mathrm{H}\right\} \mathrm{NMR}\left(400 \mathrm{MHz},\left(C D_{3}\right)_{2} C O\right)\right) \delta: 64.8(\mathrm{~s}, 1 \mathrm{P}$, $\left.P P h_{2}\right), 41.4\left(\right.$ s, $\left.1 \mathrm{P}, P P h_{2}\right)$. 
${ }^{1} \mathrm{H}$ NMR (400 MHz, DMSO) $\delta: 9.19(\mathrm{~s}, 1 \mathrm{H}, N H), 8.73\left(\mathrm{~m}, 1 \mathrm{H}, H_{6}\right), 7.86\left(\mathrm{~m}, 1 \mathrm{H}, H_{4}\right)$, $7.57\left(\mathrm{~m}, 24 \mathrm{H}, H_{P h}\right), 7.03\left(\mathrm{~d}, 1 \mathrm{H}, H_{3},{ }^{3} J_{H H} 8.3 \mathrm{~Hz}\right), 6.86\left(\mathrm{~m}, 1 \mathrm{H}, H_{5}\right) .{ }^{19} \mathrm{~F}$ NMR $(400$ $\left.\mathrm{MHz},\left(C D_{3}\right)_{2} C O\right) \delta:-80.05$ (s, 1F, OTf). ${ }^{31} \mathrm{P}\left\{{ }^{1} \mathrm{H}\right\} \mathrm{NMR}\left(400 \mathrm{MHz},\left(C D_{3}\right)_{2} C O\right) \delta: 64.9$ (s br, 1P, $\left.P P h_{2}\right), 37.4\left(\mathrm{~s}, 1 \mathrm{P}, P P h_{3}\right) .{ }^{13} \mathrm{C}\left\{{ }^{1} \mathrm{H}\right\} \mathrm{NMR}(300 \mathrm{MHz}, D M S O) \delta: 155.61(\mathrm{~s}, 1 \mathrm{C}$, $\left.C_{2}\right), 151.69\left(\mathrm{~m}, 1 \mathrm{C}, C_{6^{\prime}}\right), 147.50\left(\mathrm{~s}, 1 \mathrm{C}, C_{6}\right), 139.03\left(\mathrm{~s}, 1 \mathrm{C}, C_{4}\right), 137.76\left(\mathrm{~s}, 1 \mathrm{C}, C_{4^{\prime}}\right)$, 134.54 (d, 10C, $\left.C_{\text {orthoPPh, }}{ }^{3} J_{H H} 10.4 \mathrm{~Hz}\right), 132.61$ (s, 10C, $\left.C_{\text {paraPh }}\right), 132.33$ (s, 1C, $\left.C_{3}{ }^{\prime}\right)$, $129.80\left(\mathrm{~s}, 5 \mathrm{C}, C_{\text {metaph }}\right), 125.97\left(\mathrm{~s}, 1 \mathrm{C}, C_{5}\right), 117.00\left(\mathrm{~s}, 1 \mathrm{C}, C_{5}\right), 112.77\left(\mathrm{~s}, 1 \mathrm{C}, C_{3}\right) . \mathrm{MS}$ $(\mathrm{ESI}+): \mathrm{m} / \mathrm{z}(\%)=738.2\left([\mathrm{M}-\mathrm{OTf}]^{+}, 100\right), 723.2\left(\left[\left(\mathrm{PPh}_{2} \mathrm{py}\right)_{2} \mathrm{Au}\right]^{+}, 49.4\right), 753.2$ $\left(\left[\left(\mathrm{PPh}_{2} \mathrm{NHpy}\right)_{2} \mathrm{Au}\right]^{+}, 25.8\right)$.

Synthesis of $\left[\mathrm{Au}\left(\mathrm{PPh}_{2} \mathrm{NHpy}\right)_{2}\right](\mathrm{OTf})$ (5)

To a solution of L1 (0.055 g, $0.2 \mathrm{mmol})$ in $20 \mathrm{~mL}$ of dichloromethane was added $\left[\mathrm{Au}(\mathrm{tht})_{2}\right](\mathrm{OTf})(0.057 \mathrm{~g}, 0.11 \mathrm{mmol})$. The mixture was stirred for $2 \mathrm{~h}$ and then evaporation of the solvent to $c a .5 \mathrm{~mL}$ and addition of hexane gave complex 5 as a white solids. Yield: $0.077 \mathrm{~g}, 78.25 \%$. $\mathrm{C}_{35} \mathrm{H}_{30} \mathrm{AuF}_{3} \mathrm{~N}_{4} \mathrm{O}_{3} \mathrm{P}_{2} \mathrm{~S}$ (902.61): calcd. C, 46.57; $\mathrm{H}, 3.35 ; \mathrm{N}, 6.21 ; \mathrm{S}, 3.55$, found $\mathrm{C}, 46.32 ; \mathrm{H}, 3.52 ; \mathrm{N}, 6.11 ; \mathrm{S}, 3.91 . \mathrm{IR}\left(\mathrm{cm}^{-1}\right): v(\mathrm{NH})=$ $3243, v(\mathrm{C}=\mathrm{N})=1591, v(\mathrm{PN})=901, v_{\text {asym }}\left(\mathrm{SO}_{3}\right)=1258, v_{\text {sym }}\left(\mathrm{CF}_{3}\right)=1218, v_{\text {asym }}\left(\mathrm{CF}_{3}\right)=$ $1166, v_{\text {sym }}\left(\mathrm{SO}_{3}\right)=1027 .{ }^{1} \mathrm{H}$ NMR (400 MHz, DMSO) $\delta: 8.95$ (s br, $\left.1 \mathrm{H}, N H\right), 7.75$ (m, 1 $\left.\mathrm{H}, H_{6}\right), 7.54\left(\mathrm{~m}, 1 \mathrm{H}, 11 \mathrm{H}, H_{P h, y}\right), 6.98\left(\mathrm{~m}, 1 \mathrm{H}, H_{3}\right), 6.78\left(\mathrm{~m}, 1 \mathrm{H}, H_{5}\right) .{ }^{19} \mathrm{~F}$ NMR (400 MHz, DMSO) $\delta:-77.72$ (s, 1F, OTf). ${ }^{31} \mathrm{P}\left\{{ }^{1} \mathrm{H}\right\}$ NMR (400 MHz, DMSO) $\delta: 62.3$ (s, 1P, $\left.P P h_{2}\right) .{ }^{13} \mathrm{C}\left\{{ }^{1} \mathrm{H}\right\}$ NMR (400 MHz, DMSO) $\delta: 156.00$ (d, $\left.1 \mathrm{C}, C_{2},{ }^{2} J_{\mathrm{CP}} 3.5 \mathrm{~Hz}\right), 146.76(\mathrm{~s}$, $\left.1 \mathrm{C}, C_{6}\right), 137.56\left(\mathrm{~s}, 1 \mathrm{C}, C_{4}\right), 135.55\left(\mathrm{~d}, 1 \mathrm{C}, C_{i p s o P h},{ }^{1} J_{\mathrm{CP}} 37.0 \mathrm{~Hz}\right), 131.63(\mathrm{~d}, 1 \mathrm{C}$, $\left.C_{\text {orthoPh }},{ }^{2} J_{\mathrm{CP}} 17.0 \mathrm{~Hz}\right), 130.44$ (s, $\left.1 \mathrm{C}, C_{\text {paraPh }}\right), 128.36$ (d, $\left.1 \mathrm{C}, C_{\text {metaPh }},{ }^{3} J_{\mathrm{CP}} 9.7 \mathrm{~Hz}\right)$, $115.22\left(\mathrm{~s}, 1 \mathrm{C}, C_{5}\right), 111.74\left(\mathrm{~d}, 1 \mathrm{C}, C_{3},{ }^{3} J_{\mathrm{CP}} 2.4 \mathrm{~Hz}\right)$. MS (ESI+): m/z (\%) = $753.2([\mathrm{M}-$ $\left.\mathrm{OTf}^{+}, 100\right)$.

\section{Synthesis of $\left[\mathrm{Ag}\left(\mathrm{PPh}_{2} \mathrm{NHpy}\right)_{2}\right](\mathrm{OTf})(6)$}

To a solution of L1 (0.055 g, $0.2 \mathrm{mmol})$ in $20 \mathrm{~mL}$ of dichloromethane was added $\operatorname{Ag}(\mathrm{OTf})(0.025 \mathrm{~g}, 0.1 \mathrm{mmol})$ or $[\mathrm{Ag}(\mathrm{tht})(\mathrm{OTf})](0.034 \mathrm{~g}, 0.1 \mathrm{mmol}$.). The mixture was stirred for $1 \mathrm{~h}$ and then evaporation of the solvent to $c a .5 \mathrm{~mL}$ and addition of hexane gave complex 6 as a white solid. Yield: $0.134 \mathrm{~g}, 82.72 \% . \mathrm{C}_{33} \mathrm{H}_{30} \mathrm{AgF}_{3} \mathrm{~N}_{4} \mathrm{O}_{3} \mathrm{P}_{2} \mathrm{~S}$ (813.51): calcd. C, 51.67; H, 3.72; N, 6.89; S, 3.94, found C, 51.45; H, 3.89; N, 6.90; S, 
4.20. IR $\left(\mathrm{cm}^{-1}\right): v(\mathrm{NH})=3243, v(\mathrm{C}=\mathrm{N})=1592, v(\mathrm{PN})=902, v_{\text {asym }}\left(\mathrm{SO}_{3}\right)=1273$ y 1250 , $v_{\text {sym }}\left(\mathrm{CF}_{3}\right)=1218, v_{\text {asym }}\left(\mathrm{CF}_{3}\right)=1152, v_{\text {sym }}\left(\mathrm{SO}_{3}\right)=1029 .{ }^{1} \mathrm{H} \mathrm{NMR}\left(400 \mathrm{MHz},\left(C D_{3}\right)_{2} \mathrm{CO}\right)$ $\delta: 7.72\left(\mathrm{~m}, 2 \mathrm{H}, N H\right.$ у $\left.H_{6}\right), 7.62\left(\mathrm{~m}, 1 \mathrm{H}, H_{4}\right), 7.47\left(\mathrm{~m}, 6 \mathrm{H}, H_{P h}\right), 7.34\left(\mathrm{~m}, 4 \mathrm{H}, H_{P h}\right), 7.04$ $\left(\mathrm{d}, 1 \mathrm{H}, H_{3},{ }^{3} J_{\mathrm{HH}} 8.3 \mathrm{~Hz}\right), 6.90\left(\mathrm{~m}, 1 \mathrm{H}, H_{5}\right) .{ }^{19} \mathrm{~F} \mathrm{NMR}\left(400 \mathrm{MHz},\left(C D_{3}\right)_{2} C O\right) \delta:-80.07(\mathrm{~s}$, 1F, OTf). ${ }^{31} \mathrm{P}\left\{{ }^{1} \mathrm{H}\right\}$ NMR (400 MHz, $\left.\left(C D_{3}\right)_{2} C O\right) \delta: 29.9$ (s, $\left.1 \mathrm{P}, P P h_{2}\right) .{ }^{31} \mathrm{P}\left\{{ }^{1} \mathrm{H}\right\} \mathrm{NMR}$, $\left(400 \mathrm{MHz},\left(C D_{3}\right)_{2} C O,-70^{\circ} \mathrm{C}\right) \delta: 30.1\left(2 \mathrm{~d}, 2 \mathrm{P}, P P h_{2}, J_{109 \mathrm{Ag}-\mathrm{P}} 398.8 \mathrm{~Hz}, J_{107 \mathrm{Ag}-\mathrm{P}} 347.5 \mathrm{~Hz}\right.$ ). ${ }^{13} \mathrm{C}\left\{{ }^{1} \mathrm{H}\right\}$ NMR (400 MHz, DMSO) $\delta: 156.63$ (d, $\left.1 \mathrm{C}, C_{2},{ }^{2} J_{\mathrm{CP}} 8.1 \mathrm{~Hz}\right), 147.14$ (s, $1 \mathrm{C}$, $\left.C_{6}\right), 138.67\left(\mathrm{~s}, 1 \mathrm{C}, C_{4}\right), 134.90\left(\mathrm{~d}, 1 \mathrm{C}, C_{\mathrm{i} p s o P h},{ }^{1} J_{\mathrm{CP}} 25.1 \mathrm{~Hz}\right), 131.36\left(\mathrm{~d}, 1 \mathrm{C}, C_{o r t h o P h}\right.$ $\left.{ }^{2} J_{\mathrm{CP}} 18.9 \mathrm{~Hz}\right), 130.41$ (s, $\left.1 \mathrm{C}, C_{\text {paraph }}\right), 128.66$ (d, $\left.1 \mathrm{C}, C_{\text {metaPh }},{ }^{3} J_{\mathrm{CP}} 9.5 \mathrm{~Hz}\right), 115.44(\mathrm{~s}, 1$ $\left.\mathrm{C}, C_{5}\right), 112.29\left(\mathrm{~s}, 1 \mathrm{C}, C_{3}\right)$. MS $(\mathrm{ESI}+): \mathrm{m} / \mathrm{z}(\%)=665.0\left([\mathrm{M}-\mathrm{OTf}]^{+}, 100\right)$.

\section{Synthesis of $\left[\mathrm{Ag}\left(\mathrm{PPh}_{2} \mathrm{NHpy}\right)(\mathrm{OTf})\right](7)$}

To a solution of L1 (0.055 g, $0.2 \mathrm{mmol})$ in $25 \mathrm{~mL}$ of dichloromethane was added $\mathrm{Ag}(\mathrm{OTf})(0.051 \mathrm{~g}, 0.2 \mathrm{mmol})$. The mixture was stirred for $1 \mathrm{~h}$ and then evaporation of the solvent to $c a .5 \mathrm{~mL}$ and addition of hexane gave complex 7 as a white solid. Yield: 0.083 g, $77.80 \%$. $\mathrm{C}_{18} \mathrm{H}_{15} \mathrm{AgF}_{3} \mathrm{~N}_{2} \mathrm{O}_{3} \mathrm{PS}$ (535.23): calcd. C, 40.39; H, 2.82; N, 5.23; S, 5.99, found C, 40.39; H, 2.92; N, 2.88; S, 5.71. IR $\left(\mathrm{cm}^{-1}\right): v(\mathrm{NH})=3257, v(\mathrm{C}=\mathrm{N})=$ $1591, v(\mathrm{PN})=916 v_{\text {asym }}\left(\mathrm{SO}_{3}\right)=1273$ y $1241, v_{\text {sym }}\left(\mathrm{CF}_{3}\right)=1222, v_{\text {asym }}\left(\mathrm{CF}_{3}\right)=1155, \mathrm{y}$ $v_{\text {sym }}\left(\mathrm{SO}_{3}\right)=1024 .{ }^{1} \mathrm{H}$ NMR $\left(400 \mathrm{MHz},\left(C D_{3}\right)_{2} C O\right) \delta: 8.24(\mathrm{~s} \mathrm{br}, 1 \mathrm{H}, N H), 7.93(\mathrm{~m}, 1 \mathrm{H}$, $\left.H_{6}\right), 7.76\left(\mathrm{~m}, 5 \mathrm{H}, H_{P h}\right), 7.55\left(\mathrm{~m}, 6 \mathrm{H}, H_{P h}\right.$ y $), 7.18\left(\mathrm{~m}, 1 \mathrm{H}, H_{3}\right), 6.90\left(\mathrm{~m}, 1 \mathrm{H}, H_{5}\right) .{ }^{19} \mathrm{~F}$ NMR $\left(400 \mathrm{MHz},\left(C D_{3}\right){ }_{2} C O\right) \delta:-80.00$ (s, $\left.1 \mathrm{~F}, \mathrm{OTf}\right) .{ }^{31} \mathrm{P}\left\{{ }^{1} \mathrm{H}\right\}$ NMR (400 MHz, $\left.\left.\left(C D_{3}\right)_{2} C O\right)\right) \delta: 25.4\left(\mathrm{~s}, 1 \mathrm{P}, P P h_{2}\right) .{ }^{31} \mathrm{P}\left\{{ }^{1} \mathrm{H}\right\} \mathrm{NMR},\left(300 \mathrm{MHz},\left(C D C l_{3}\right),-70^{\circ} \mathrm{C}\right) \delta: 24.8$ $\left(2 \mathrm{~d}, 1 \mathrm{P}, P P h_{2}, J_{109 \mathrm{Ag}-\mathrm{P}} 493.9, J_{107 \mathrm{Ag}-\mathrm{P}} 427.9\right) .{ }^{13} \mathrm{C}\left\{{ }^{1} \mathrm{H}\right\} \mathrm{NMR}\left(400 \mathrm{MHz},\left(C D_{3}\right)_{2} C O\right) \delta$ : 158.37 (s br, $1 \mathrm{C}, C_{2}$ ), 149.37 (s br, 1 C, $C_{6}$ ), 141.91 (s br, 1 C, $C_{4}$ ), 135.85 (d, 1 C, $\left.C_{\text {ipsoPh }},{ }^{1} J_{\mathrm{CP}} 28.3 \mathrm{~Hz}\right), 133.73$ (d ,6 C, $\left.C_{\text {orthoPh }},{ }^{2} J_{\mathrm{CP}} 19.3 \mathrm{~Hz}\right), 130.09$ (s, $3 \mathrm{C}, C_{\text {paraPh }}$ ), $130.02\left(\mathrm{~s}, 6 \mathrm{C}, C_{\text {metaPh }}{ }^{3} J_{\mathrm{CP}} 10.5 \mathrm{~Hz}\right), 118.37$ (s, $\left.1 \mathrm{C}, C_{5}\right), 115.20$ (s br, $\left.1 \mathrm{C}, C_{3}\right)$.

Synthesis of $\left[\mathrm{Ag}\left(\mathrm{PPh}_{3}\right)_{n}\left(\mathrm{PPh}_{2} \mathrm{NHpy}\right)\right](\mathrm{OTf})(n=1$ (8), $n=2$ (9))

To a solution of L1 (0.055 g, $0.2 \mathrm{mmol})$ in $20 \mathrm{~mL}$ of dichloromethane was added $\left[\mathrm{Ag}(\mathrm{OTf})\left(\mathrm{PPh}_{3}\right)\right]\left(0.104 \mathrm{~g}, 0.2 \mathrm{mmol}\right.$.) or $\left[\mathrm{Ag}(\mathrm{OTf})\left(\mathrm{PPh}_{3}\right)_{2}\right](0.156 \mathrm{~g}, 0.2 \mathrm{mmol}$.). The mixture was stirred for $1 \mathrm{~h}$ and then evaporation of the solvent to $c a .5 \mathrm{~mL}$ and addition of hexane gave complexes $\mathbf{8}$ and $\mathbf{9}$ as white solid. 
Complex 8. Yield: 0.129 g, $81.10 \% . \mathrm{C}_{36} \mathrm{H}_{30} \mathrm{AgF}_{3} \mathrm{~N}_{2} \mathrm{O}_{3} \mathrm{P}_{2} \mathrm{~S}$ (797.51): calcd. C, 54.22; $\mathrm{H}$, 3.79; N, 5.31; S, 4.02, found C, 54.71; H, 3.92; N, 5.41; S, 4.22. IR $\left(\mathrm{cm}^{-1}\right): v(\mathrm{NH})=$ 3223, $v(\mathrm{C}=\mathrm{N})=1599, v(\mathrm{PN})=906, v_{\mathrm{asym}}\left(\mathrm{SO}_{3}\right)=1282$ y $1243, v_{\mathrm{sym}}\left(\mathrm{CF}_{3}\right)=1218$, $v_{\text {asym }}\left(\mathrm{CF}_{3}\right)=1152, v_{\text {sym }}\left(\mathrm{SO}_{3}\right)=1027 .{ }^{1} \mathrm{H}$ NMR $\left(400 \mathrm{MHz},\left(C D_{3}\right)_{2} C O\right) \delta: 8.22(\mathrm{~s} \mathrm{br}, 1 \mathrm{H}$, $N H), 7.87\left(\mathrm{~m}, 1 \mathrm{H}, H_{6}\right), 7.74\left(\mathrm{~m}, 1 \mathrm{H}, H_{4}\right), 7.41\left(\mathrm{~m}, 25 \mathrm{H}, H_{P h}\right), 7.21\left(\mathrm{~d}, 1 \mathrm{H}, H_{3},{ }^{3} J_{\mathrm{HH}} 8.3\right.$ $\mathrm{Hz}), 6.81\left(\mathrm{~m}, 1 \mathrm{H}, H_{5}\right) .{ }^{19} \mathrm{~F}$ NMR $\left(400 \mathrm{MHz},\left(C D_{3}\right)_{2} C O\right) \delta:-80.01(\mathrm{~s}, 1 \mathrm{~F}, \mathrm{OTf}) .{ }^{31} \mathrm{P}\left\{{ }^{1} \mathrm{H}\right\}$ NMR (400 MHz, $\left.\left(C D_{3}\right)_{2} C O\right) \delta: 25.6$ (s, 1P, $\left.P P h_{2}\right), 11.9$ (s, $\left.1 \mathrm{P}, P P h_{3}\right) .{ }^{31} \mathrm{P}\left\{{ }^{1} \mathrm{H}\right\} \mathrm{NMR}$ $\left.\left(300 \mathrm{MHz},\left(C D_{3}\right)_{2} \mathrm{CO}\right),-70^{\circ} \mathrm{C}\right) \delta: 24.0\left(2 \mathrm{~d}, 1 \mathrm{P}, P P h_{2}, J_{109 \mathrm{Ag}-\mathrm{P}} 490.5, J_{107 \mathrm{Ag}-\mathrm{P}} 425.8 \mathrm{~Hz}\right)$, 9.16 ( $\mathrm{AB}_{2}$ system, $\left.J_{109 \mathrm{Ag}-\mathrm{P}} 368.5, J_{107 \mathrm{Ag}-\mathrm{P}} 319.3, J_{\mathrm{AB}} 66.7 \mathrm{~Hz}\right) .{ }^{13} \mathrm{C}\left\{{ }^{1} \mathrm{H}\right\} \mathrm{NMR}(300 \mathrm{MHz}$, DMSO) $\delta: 156.38$ (s br, $1 \mathrm{C}, C_{2}$ ), 146.98 (s, $1 \mathrm{C}, C_{6}$ ), 138.89 (s, $\left.1 \mathrm{C}, C_{4}\right), 134.18$ (d, $1 \mathrm{C}$, $\left.C_{\text {ipsoPh }},{ }^{1} J_{\mathrm{CP}} 26.8 \mathrm{~Hz}\right), 132.66$ (d, $\left.1 \mathrm{C}, C_{\text {orthoPh }},{ }^{2} J_{\mathrm{CP}} 16.4 \mathrm{~Hz}\right), 131.14$ (s, $\left.1 \mathrm{C}, C_{\mathrm{ipsoPh}}\right)$, 130.81 (d, $\left.1 \mathrm{C}, C_{\text {orthoPh }},{ }^{2} J_{\mathrm{CP}} 18.4 \mathrm{~Hz}\right), 130.15$ (s, $1 \mathrm{C}, C_{\text {paraph }}$ ), 128.61 (d, $1 \mathrm{C}, C_{\text {metaPh }}$, $\left.{ }^{3} J_{\mathrm{CP}} 9.3 \mathrm{~Hz}\right), 128.37$ (d, $\left.1 \mathrm{C}, C_{\text {metaPh }},{ }^{3} J_{\mathrm{CP}} 6.7 \mathrm{~Hz}\right), 115.44$ (s, $\left.1 \mathrm{C}, C_{5}\right), 112.27$ (s, $1 \mathrm{C}$, $\left.C_{3}\right)$. MS (ESI+): m/z (\%) $=647.00\left([\mathrm{M}-\mathrm{OTf}]^{+}, 100\right)$.

Complex 9. Yield: 0.179 g, $84.80 \% . \mathrm{C}_{54} \mathrm{H}_{45} \mathrm{AgF}_{3} \mathrm{~N}_{2} \mathrm{O}_{3} \mathrm{P}_{3} \mathrm{~S}$ (1059.80): calcd. C, 61.20; $\mathrm{H}$, 4.28; N, 2.64; S, 3.03, found C, 61.09; H, 4.30; N, 2.71; S, 3.46. IR $\left(\mathrm{cm}^{-1}\right): v(\mathrm{NH})=$ $3199, v_{\text {asym }}\left(\mathrm{SO}_{3}\right)=1289$ у $1244, v_{\text {sym }}\left(\mathrm{CF}_{3}\right)=1222, v(\mathrm{C}=\mathrm{N})=1599, v(\mathrm{PN})=907$, $v_{\text {asym }}\left(\mathrm{CF}_{3}\right)=1152, v_{\text {sym }}\left(\mathrm{SO}_{3}\right)=1026 .{ }^{1} \mathrm{H}$ NMR $\left(300 \mathrm{MHz},\left(C D_{3}\right)_{2} C O\right) \delta: 7.97(\mathrm{~s} \mathrm{br}, 1 \mathrm{H}$, $N H), 7.82\left(\mathrm{~m}, 1 \mathrm{H}, H_{6}\right), 7.67\left(\mathrm{~m}, 1 \mathrm{H}, H_{4}\right), 7.44\left(\mathrm{~m}, 4 \mathrm{H}, H_{P h}\right), 7.11\left(\mathrm{~m}, 27 \mathrm{H}, H_{3}\right.$ y $\left.P h\right)$, $6.71\left(\mathrm{~m}, 1 \mathrm{H}, H_{5}\right) .{ }^{19} \mathrm{~F}$ NMR $\left(400 \mathrm{MHz},\left(C_{3}\right){ }_{2} \mathrm{CO}\right) \delta:-80.07$ (s, 1F, OTf). ${ }^{19} \mathrm{~F}$ NMR (400 $\left.\mathrm{MHz},\left(C D_{3}\right)_{2} C O\right) \delta:-80.0$ (s, 1F, OTf). ${ }^{31} \mathrm{P}\left\{{ }^{1} \mathrm{H}\right\}$ NMR $\left(300 \mathrm{MHz},\left(C D_{3}\right)_{2} C O\right) \delta: 26.2$ (s, 1P, $\left.P P h_{2}\right), 8.5\left(\mathrm{~s}, 2 \mathrm{P}, P P h_{3}\right) .{ }^{31} \mathrm{P}\left\{{ }^{1} \mathrm{H}\right\}$ NMR $\left(300 \mathrm{MHz},\left(C D_{3}\right)_{2} C O,-70^{\circ} \mathrm{C}\right) \delta: 9.9$ y 9.3 (2d), 23.9 у $21.8(2 \mathrm{~m})$, ( $\mathrm{AB}_{2}$ system, $\left.J_{109 \mathrm{Ag}-\mathrm{P}} 374.4, J_{107 \mathrm{Ag}-\mathrm{P}} 324.3, J_{\mathrm{AB}} 66.3 \mathrm{~Hz}\right)$. ${ }^{13} \mathrm{C}\left\{{ }^{1} \mathrm{H}\right\}$ NMR $\left(300 \mathrm{MHz},\left(C D_{3}\right)_{2} C O\right) \delta: 159.16$ (s br, $\left.1 \mathrm{C}, C_{2}\right), 149.79$ (s, $\left.1 \mathrm{C}, C_{6}\right)$, 141.13 (s, $\left.1 \mathrm{C}, C_{4}\right), 137.19$ (d, $\left.1 \mathrm{C}, C_{\text {ipsoPh }},{ }^{1} J_{\mathrm{CP}} 22.5 \mathrm{~Hz}\right), 135.28$ (d, $1 \mathrm{C}, C_{\text {orthoPh }},{ }^{2} J_{\mathrm{CP}}$ $16.3 \mathrm{~Hz}), 134.20\left(1 \mathrm{C}, C_{\mathrm{i} p s o P h},{ }^{1} J_{\mathrm{CP}} 23.2 \mathrm{~Hz}\right), 133.13$ (d, $\left.1 \mathrm{C}, C_{\text {orthoPh }},{ }^{2} J_{\mathrm{CP}} 18.4 \mathrm{~Hz}\right)$, 132.28 (s, $\left.1 \mathrm{C}, C_{\text {paraPh }}\right), 132.20$ (s, $\left.1 \mathrm{C}, C_{\text {paraPh }}\right), 130.90$ (d, $\left.1 \mathrm{C}, C_{\text {metaPh }},{ }^{3} J_{\mathrm{CP}} 9.2 \mathrm{~Hz}\right)$, 130.59 (d, $\left.1 \mathrm{C}, C_{\text {metaPh }},{ }^{3} J_{\mathrm{CP}} 9.3 \mathrm{~Hz}\right), 118.02$ (s, $\left.1 \mathrm{C}, C_{5}\right), 114.85$ (s, $\left.1 \mathrm{C}, C_{3}\right) . \mathrm{MS}$ $(\mathrm{ESI}+): \mathrm{m} / \mathrm{z}(\%)=647.09\left([\mathrm{M}-\mathrm{OTf}]^{+}, 84.1\right)$. 
To a solution of L2 (0.053 g, $0.2 \mathrm{mmol})$ in $20 \mathrm{~mL}$ of dichloromethane was added $\left[\mathrm{Au}\left(\mathrm{C}_{6} \mathrm{~F}_{5}\right)(\mathrm{tht})\right](0.090 \mathrm{~g}, 0.2 \mathrm{mmol})$ The mixture was stirred for $1 \mathrm{~h}$ and then evaporation of the solvent to $c a .5 \mathrm{~mL}$ and addition of hexane gave complex $\mathbf{1 1}$ as a white solids. Yield: 0.092 g, $73.21 \% . \mathrm{C}_{20} \mathrm{H}_{13} \mathrm{AuF}_{5} \mathrm{~N}_{4} \mathrm{P}$ (632.28): calcd. C, 37.99; H, $2.07 ; \mathrm{N}, 8.86$, found $\mathrm{C}, 38.31 ; \mathrm{H}, 2.43 ; \mathrm{N}, 8.93$. IR $\left(\mathrm{cm}^{-1}\right): v(\mathrm{NH})=3231, v(\mathrm{C}=\mathrm{N})=$ $1550, v(\mathrm{PN})=905, v\left(\mathrm{C}_{6} \mathrm{~F}_{5}\right)=1500,953,793 .{ }^{1} \mathrm{H}$ NMR $(400 \mathrm{MHz}, D M S O) \delta: 13.41(\mathrm{~s}$ br, 1H, NHtrz), 9.04 (s br,1H, NH-P), 8.25 (s br, 1H, $H_{5}$ ), 7.77 (m, 4H, $\left.H_{P h}\right), 7.59$ (m, $\left.6 \mathrm{H}, H_{P h}\right) .{ }^{31} \mathrm{P}\left\{{ }^{1} \mathrm{H}\right\}$ NMR (400 MHz, DMSO) $\delta: 68.9$ (s, 1P, $\left.P P h_{2}\right) .{ }^{19} \mathrm{~F}$ NMR (400 MHz, DMSO) $\delta:(-114.26)-(-114.82)\left(\mathrm{m}, 2 \mathrm{~F}, F_{\text {ortho }}\right),-158.43\left(\mathrm{t}, 1 \mathrm{~F}, F_{\text {para }}, J_{\text {meta-para }}=21.21\right.$ $\mathrm{Hz},),(-161.51)-(-161.99)\left(\mathrm{m}, 2 \mathrm{~F}, F_{\text {meta }}\right)$. MS (ESI+): m/z (\%) $=632.9\left([\mathrm{M}+\mathrm{H}]^{+}, 31.8\right)$.

Synthesis of $\left[\mathrm{Au}\left\{\mathrm{PPh}_{2} \mathrm{NH}(\mathrm{Htrz})\right\}_{2}\right](\mathrm{OTf})(\mathbf{1 2})$

To a solution of L2 $(0.053 \mathrm{~g}, 0.2 \mathrm{mmol})$ in $20 \mathrm{~mL}$ of dichloromethane was added $\left[\mathrm{Au}(\mathrm{tht})_{2}\right](\mathrm{OTf})(0.057 \mathrm{~g}, 0.11 \mathrm{mmol})$. The mixture was stirred for $2 \mathrm{~h}$ and then evaporation of the solvent to ca. $5 \mathrm{~mL}$ and addition of hexane gave complex 12 as a white solids. Yield: 0.070 g, $79.86 \% \mathrm{C}_{29} \mathrm{H}_{26} \mathrm{AuF}_{3} \mathrm{~N}_{8} \mathrm{O}_{3} \mathrm{P}_{2} \mathrm{~S}$ (882.54): calcd. C, 39.47; $\mathrm{H}$, 2.97; N, 12.70; S, 3.63, found C, 39.31; H, 2.98; N, 12.89; S, 3.44. IR $\left(\mathrm{cm}^{-1}\right): v(\mathrm{NH})=$ $3313, v(\mathrm{C}=\mathrm{N})=1585, v(\mathrm{PN})=1024, v_{\mathrm{asym}}\left(\mathrm{SO}_{3}\right)=1288$ y $1238, v_{\mathrm{sym}}\left(\mathrm{CF}_{3}\right)=1220$, $v_{\text {asym }}\left(\mathrm{CF}_{3}\right)=1096, v_{\text {sym }}\left(\mathrm{SO}_{3}\right)=1024 .{ }^{1} \mathrm{H} \mathrm{NMR}(400 \mathrm{MHz}, D M S O) \delta: 13.55$ (s br, $1 \mathrm{H}$, NHtrz), 9.37 (s br,1H, NH-P), 8.33 (m, 1H, $\left.H_{5}\right), 7.77$ (m, 4 H, $\left.H_{P h}\right), 7.60$ (m, 7H, $\left.H_{P h}\right)$. ${ }^{19} \mathrm{~F}\left\{{ }^{1} \mathrm{H}\right\}$ NMR $\left.\left(400 \mathrm{MHz},\left(C D_{3}\right){ }_{2} C O\right)\right) \delta:-77.7$ (s, 1F, OTf). ${ }^{31} \mathrm{P}\left\{{ }^{1} \mathrm{H}\right\}$ NMR $(400 \mathrm{MHz}$, DMSO) $\delta: 68.7$ (s, 1P, $\left.P P h_{2}\right)$. MS (ESI+): $\mathrm{m} / \mathrm{z}(\%)=733.2\left([\mathrm{M}-\mathrm{OTf}]^{+}, 100\right)$.

Synthesis of $\left.\mathrm{Ag}\left\{\mathrm{PPh}_{2} \mathrm{NH}(\mathrm{Htrz})\right\}\right](\mathrm{OTf})(\mathbf{1 3})$ and $\left[\mathrm{Ag}\left\{\mathrm{PPh}_{2} \mathrm{NH}(\mathrm{Htrz})\right\}_{2}\right](\mathrm{OTf})(\mathbf{1 4})$.

To a solution of L2 $(0.053 \mathrm{~g}, 0.2 \mathrm{mmol})$ in $20 \mathrm{~mL}$ of dichloromethane was added $\operatorname{Ag}(\mathrm{OTf})(0.025 \mathrm{~g}, 0.1 \mathrm{mmol}$ or $0.05 \mathrm{~g}, 0.2 \mathrm{mmol})$ or [Ag(tht)(OTf)] $(0.069 \mathrm{~g}, 0.2$ mmol). The mixture was stirred for $1 \mathrm{~h}$ and then evaporation of the solvent to $c a .5 \mathrm{~mL}$ and addition of hexane gave complexes 13 and 14 as white solids. 
Complex 13. Yield: 0.076 g, $73.20 \%$. $\mathrm{C}_{15} \mathrm{H}_{13} \mathrm{AgF}_{3} \mathrm{~N}_{4} \mathrm{O}_{3} \mathrm{PS}$ (525.19): calcd. C, 34.30; H, 2.49; N, 10.67; S, 6.11, found C, 34.77; H, 2,49; N, 10.83, S, 6.42. IR $\left(\mathrm{cm}^{-1}\right): v(\mathrm{NH})=$ $3207, v(\mathrm{C}=\mathrm{N})=1570, v(\mathrm{PN})=932, v_{\text {asym }}\left(\mathrm{SO}_{3}\right)=1272$ y $1243, v_{\mathrm{sym}}\left(\mathrm{CF}_{3}\right)=1223, v\left(\mathrm{CF}_{3}\right)$ $=1024, v_{\mathrm{sym}}\left(\mathrm{SO}_{3}\right)=981 .{ }^{1} \mathrm{H} \mathrm{NMR}(400 \mathrm{MHz}, D M S O) \delta: 13.63$ (s br, 1H, NHtrz), 8.80 (s br,1H, NH-P), 8.44 (s br, 1H, $\left.H_{5}\right), 7.52\left(\mathrm{~m}, 10 \mathrm{H}, H_{P h}\right) .{ }^{19} \mathrm{~F}$ NMR (400 MHz, DMSO) $\delta:-77.73$ (s, 1F, OTf). $\left.{ }^{31} \mathrm{P}\left\{{ }^{1} \mathrm{H}\right\} \mathrm{NMR}\left(400 \mathrm{MHz},\left(C D_{3}\right)_{2} C O\right)\right) \delta: 37.3\left(\mathrm{~s}, 1 \mathrm{P}, P P h_{2}\right)$.

Complex 14. Yield: 0.060 g, $76.0 \%$. $\mathrm{C}_{29} \mathrm{H}_{26} \mathrm{AgF}_{3} \mathrm{~N}_{8} \mathrm{O}_{3} \mathrm{P}_{2} \mathrm{~S}$ (793.44): calcd. C, 43.90; H, 3.30; N, 14.12; S, 4.04, found C, 43.62; H, 3.29; N, 14.47, S, 4.38. IR $\left(\mathrm{cm}^{-1}\right): v(\mathrm{NH})=$ $3180, v(\mathrm{C}=\mathrm{N})=1565, v(\mathrm{PN})=922, v_{\text {asym }}\left(\mathrm{SO}_{3}\right)=1276$ y $1234, v_{\text {sym }}\left(\mathrm{CF}_{3}\right)=1170, v\left(\mathrm{CF}_{3}\right)$ $=1100, v_{\text {sym }}\left(\mathrm{SO}_{3}\right)=1029 .{ }^{1} \mathrm{H}$ NMR $(400 \mathrm{MHz}, D M S O) \delta: 13.70$ (s br, 1H, NHtrz), 9.08 (s br,1H, NH-P), 8.45 (s br, 1H, $\left.H_{5}\right), 7.52\left(\mathrm{~m}, 10 \mathrm{H}, H_{P h}\right) .{ }^{19} \mathrm{~F}$ NMR (400 MHz, DMSO) $\delta:-80.31$ (s, 1F, OTf). ${ }^{31} \mathrm{P}\left\{{ }^{1} \mathrm{H}\right\}$ NMR $(400 \mathrm{MHz}, D M S O) \delta: 40.8\left(\mathrm{~s}, 2 \mathrm{P}, P P h_{2}\right) .{ }^{31} \mathrm{P}\left\{{ }^{1} \mathrm{H}\right\}$ NMR $\left(300 \mathrm{MHz}, C D C l_{3},-70^{\circ} \mathrm{C}\right) \delta: 38.6\left(2 \mathrm{~m}, 2 \mathrm{P}, P P h_{2}, J_{109 \mathrm{Ag}-\mathrm{P}} 472.2 \mathrm{~Hz}, J_{107 \mathrm{Ag}-\mathrm{P}} 413.4\right.$ Hz). MS (ESI+): m/z (\%)= $644.3\left([\mathrm{M}-\mathrm{OTf}]^{+}, 31.6\right)$.

\subsubsection{Crystal Structure Determinations}

Data were recorded with a Bruker Smart 1000 CCD (6) or Oxford Diffraction Xcalibur (9) diffractometer. The crystals were mounted in inert oil on glass fibres and transferred to the cold gas stream of the diffractometer. Data were collected by using monochromated Mo-Ka radiation $(\lambda=0.71073 \AA)$ in $\omega$-scans. Absorption corrections based on multiples cans were applied by using the SADABS program [63] for complex 6 and using spherical harmonics implemented in SCALE3 ABSPACK scaling algorithm for complex 9.The structures were solved by direct methods and refined on $F 2$ by using the SHELXL-97 program [64]. All non-hydrogen atoms were refined anisotropically. Hydrogen atoms were included by using a riding model. Further crystal data are given in Table 4. CCDC-1027814 (6) and 1027815 (9) contains the supplementary crystallographic data for this paper. These data can be obtained free of charge from The Cambridge Crystallographic Data Centre via www.ccdc.cam.ac.uk/data_request/cif. 


\subsection{Antibacterial assays}

Determination of bacterial growth inhibition by the disc diffusion method was performed by culturing bacteria in the appropriate solid media. LB- or BHI-agar plates were used for Gram -negative or Gram-positive bacteria respectively. After inoculation of bacterial cultures, sterile cellulose discs containing 5-fold serial dilutions of the tested compounds (or control antibiotics) were placed onto the agar. Plates were incubated at $37^{\circ} \mathrm{C}$ for 24 hours to examine growth inhibition zones. MIC values were calculated by observing bacterial growth inhibition in liquid cultures. LB or BHI liquid media was inoculated with 5-fold serial dilutions of the tested compounds and subsequently, tubes were inoculated with equal volumes of the corresponding bacteria and incubated for 24 hours at $37^{\circ} \mathrm{C}$. Culture density was determined by $\mathrm{Abs}$ at $600 \mathrm{~nm}$ and compared to control cultures in the absence of compounds. Those cultures showing inhibition of bacterial growth were plated on the appropriate solid media to determine the MBC. After 24 hours incubation at $37^{\circ} \mathrm{C}$, colony forming units were enumerated and compared to untreated control. A reduction of $99 \%$ in cfu numbers was considered as significant. 


\section{Abbreviations}

\begin{tabular}{|c|c|}
\hline $\mathrm{Ph}_{2} \mathrm{PNHpy}$ & 2-(diphenylphosphinoamino)pyridine \\
\hline $\mathrm{Ph}_{2} \mathrm{PNH}(\mathrm{Htrz})$ & 3-(diphenylphosphinoamino)-1,2,4-triazole \\
\hline Abs & Absorbance \\
\hline Amp & Ampicillin \\
\hline asym & Asymmetry \\
\hline $\mathrm{cfu}$ & Colony Forming Unit \\
\hline $\mathrm{Cm}$ & Chloramphenicol \\
\hline $\mathrm{CDCl}_{3}$ & Chloroform \\
\hline$\left(C D_{3}\right)_{2} \mathrm{CO}$ & Acetone \\
\hline ESI-MS & Electrospray Ionization-Mass Spectrometry \\
\hline Ery & Erythromycin \\
\hline $\mathrm{Gm}$ & Gentamicin \\
\hline IR & Infrared \\
\hline $\mathrm{MBC}$ & Minimum Bactericidal Concentration \\
\hline MIC & Minimum Inhibitory Concentration \\
\hline $\mathrm{Km}$ & Kanamycin \\
\hline NMR & Nuclear Magnetic Resonance \\
\hline Rif & Rifampicin \\
\hline sym & Symmetry \\
\hline Van & Vancomycin \\
\hline
\end{tabular}




\section{Acknowledgments}

Authors thank the Ministerio de Economía y Competitividad-FEDER CTQ2013-48635C2-1-P and FIS12/1970 and DGA-FSE (E77) for financial support. 


\section{References}

[1] N. E. Allen, Antiinfect. Agents Med. Chem. 9 (2010) 23-47.

[2] F. Novelli, M. Recine, F. Sparatore, C. Juliano, Farmaco (1999) 232-236.

[3] Ch. I. Yeo, J. H. Sim, Ch. H. Khoo, Z. J. Goh, K. P. Ang, Y. K. Cheah, Z. A. Fairuz, S. N. B. A. Halim, S. W. Ng, H. L. Seng, E. R. T. Tiekink, 46 Gold Bull (2013) $145-152$.

[4] R. Noguchi, A. Hara, A. Sugie, K. Nomiya, Inorg. Chem. Commun. 9 (2006) 355359.

[5] L. L. Marqués, G. Manzoni de Oliveira, E. Schulz Lang, M. M. Anraku de Campos, L.R. Soccol Gris, Inorg. Chem. Commun. 10 (2007) 1083-1087.

[6] Y. Hokai, B. Jurkowicz, J. Fernández-Gallardo, N. Zakirkhodjaev, M. Sanau, T. R. Muth, M. Contel, J. Inorg. Biochem. 138 (2014) 81-88.

[7] B. Đ. Glišić, M. I. Djuran, Dalton Trans. 43 (2014) 5950-5969.

[8] K. M. Hindi, M. J. Panzner, C. A. Tessier, C. L. Cannon, W. J. Youngs, Chem. Rev. 109 (2009) 3859.

[9] H.J. Klasen, Burns 26 (2000) 117-130.

[10] H. J. Klasen, Burns 26 (2000) 131-138.

[11] L. Kyros, C. N. Banti, N. Kourkoumelis, M. Kubicki, I. Sainis, S. K. Hadjikakou, J. Biol. Inorg. Chem. 19 (2014) 449-464.

[12] W. Streciwilk, J. Cassidy, F. Hackenberg, H. Müller-Bunz, F. Paradisi, M. Tacke, J. Organomet. Chem. 749 (2014) 88-99.

[13] M. A. M. Abu-Youssef, S. M. Soliman, V. Langer, Y. M. Gohar, A. A. Hasanen, M. A. Makhyoun, A. H. Zaky, L. R. Öhrström, Inorg. Chem. 49 (2010) 9788-9797.

[14] K. Nomiya, K. Tsuda, Y. Tanabe, H. Nagano, J. Inorg. Biochem. 69 (1998) 9-14.

[15] F. Hackenberg, M. Tacke, Dalton Trans. 43 (2014) 8144-8153. 
[16] A. Kascatan-Nebioglu, M. J. Panzner, C. A. Tessier, C. L. Cannon, W. J. Youngs, Coord. Chem. Rev. 251 (2007) 884-895.

[17] H. Nikaido, Microbiol. Mol. Biol. Rev. 67 (2003) 593-656.

[18] J. M. Pagès, C. E. James, M. Winterhalter, Nature rev. 6 (2008) 893-903.

[19] D. Marchaim, D. E. Katz, L. S. Munoz-Price, Curr. Transl. Geriatr. Exp. Gerontol. Rep. 2 (2013) 113-124.

[20] F. Allerberger, M. Wagner, Clin. Microbiol. Infect. 16 (2010) 16-23.

[21] G. Almeida, A. Morvan, R. Magalhães, I. Santos, T. Hogg, A. Leclercq, P. Teixeira, Eur. J. Clin. Microbiol. Infect. Dis. 29 (2010) 1219-1227.

[22] C. L Little, S. M Pires, I. A Gillespie, K. Grant, G. L Nichols, Foodborne Pathog. Dis. 7 (2010) 749-756.

[23] A. L. Monnier, A. Leclercq, Pathol. Biol. 57 (2009) 17-22.

[24] W. M. Scheld, D. D. Fletcher, F. N. Fink, M. A. Sande, J. Infect. Dis. 140 (1979) 287-294.

[25] V. Ferreira, M. Wiedmann, P.Teixeira, M. J. Stasiewicz, J. Food Prot. 77 (2014), $150-170$.

[26] T. J. V. Malley, M. J. Stasiewicz, Y.T. Grohn, S. Roof,;S. Warchocki, K. Nightingale, M. Wiedmann, J. Food Prot. 76 (2013) 796-811.

[27] S. S. Ratani, R. M. Siletzky, V. Dutta, S. Yildirim, J. A. Osborne, W. Lin, A. D. Hitchins, T. J. Ward, S. Kathariou, App. Environ. Microbiol. 78 (2012), 69386945 .

[28] E. G. Christensen, L. Gram, V. G. Kastbjerg, 55(9) Antimicrob. Agents Chemother. (2011) 4064-4071.

[29] L. D. Saravolatz, J. Pawlak, L. B. Johnson, Clin. Infec. Dis. 55 (2012) 582-586.

[30] C. A. Smith, M. Toth, M. Bhattacharya, H. Frase, S. B. Vakulenko, Acta Crystallogr D 70 (2014) 1561-1571. 
[31] P. Szweda, M. Schielmann, A. Frankowska, B. Kot, M. Zalewska, J. Vet. Med. Sci. 76 (2014) 355-362.

[32] S. Raju, A. K. Oli, S. A. Patil, R. Kelmani Chandrakanth, World J. Microbiol. Biotechnol. 26 (2010) 171-176.

[33] S. M. Sabra, N.A. Farag, Jordan J. Biol. Sci. 5 (2012) 79-84.

[34] S.L. Tan, H.Y. Lee, N.A. Mahyudin, Food Control 44 (2014) 203-207.

[35] H. A. Abdul-Ratha, M.A. Yarmorad, Int. J. Curr. Microbiol. App. Sci. 2 (2013) 493-500.

[36] M.F. Fillat, M. C. Gimeno, A. Laguna, E. Latorre, L. Ortego, M. D. Villacampa, Eur. J. Inorg. Chem. (2011) 1487-1495.

[37] S. M. Aucott, A. M. Z. Slawin, J. D. Woollins, J. Chem. Soc. Dalton Trans. (2000) $2559-2575$.

[38] L. Lettko, J. S. Wood, M. D. Rausch, Inorg. Chim. Acta 38 (2000) 37-44.

[39] A. Caballero, A. Guerrero, F. A. Jalon, B. R. Manzano, R. M. Claramunto, M. D. Santa Maria, C. Escolástico, J. Elguero, Inorg. Chim. Acta 347 (2003) 168-174.

[40] L. Muertterties, C. W. Alegranti, J. Am. Chem. Soc. 92 (1970) 4114-4115.

[41] E. L. Muertterties, C. W. Alegranti, J. Am. Chem. Soc. 94 (1972) 6386-6391.

[42] S. J. Berners-Price, R. J. Bowen, P. J. Harvey, P. C. Healy, G. A. Koutsantonis, J. Chem. Soc., Dalton Trans. (1998) 1743-1750.

[43] G. Francius, O. Domenech, M. P. Mingeot-Leclercq, Y. F. Dufrêne, J. Bacteriol. 190 (2008) 7904-7909.

[44] T. J. Beveridge, L. L. Graham, Microbiol. Rev. 55 (1991) 684-705.

[45] T. J. Beveridge, J. Bacteriol. 181 (1999) 4725-4733.

[46] G. Roymahapatra, S. M. Mandal, W. F. Porto, T. Samanta, S. Giri, J. Dinda, O. L. Franco, P. K. Chattaraj, Curr. Med. Chem. 19 (2012) 4184-4193.

[47] N. C. Kasuga, A. Sugie, K. Nomiya, Dalton Trans. (2004) 3732-3740. 
[48] K. Nomiya, A. Yoshizawa, K. Tsukagoshi, N. C. Kasuga, J. Inorg. Biochem. 98 (2004) 46-60.

[49] K. Nomiya, S. Takahashi, R. Noguchi, S. Nemoto, T. Takayama, M. Oda, Inorg. Chem. 39 (2000) 3301-3311.

[50] K. Nomiya, K. Tsuda, T. Sudoh; M. Oda, J. Inorg. Biochem. 68 (1997) 39-44.

[51] M. Altaf, H. Stoeckli-Evans, A. Cuin, D. N. Sato, F. R. Pavan, C. Q. F. Leite, S.Ahmad, M. Bouakka, M. Mimouni, F. Za. Khardli, T. B. Hadda, Polyhedron 62 (2013) 138-147.

[52] K. Nomiya; S. Takahashi, R.Noguchi, J. Chem. Soc., Dalton Trans. (2000) 20912097.

[53] K. Nomiya, K. Onoue, Y. Kondoh, N. C. Kasuga,H. Nagano, M. Oda, S. Sakuma, Polyhedron 14 (1995) 1359-1367.

[54] K. Nomiya, K. Tsuda, N. C. Kasuga, J. Chem. Soc., DaltonTrans. (1998) 16531659.

[55] K. Nomiya, S. Takahashi, R. Noguchi, J. Chem. Soc., Dalton Trans. (2000) 43694373.

[56] K. Nomiya, R. Noguchil, T. Shigeta, Y. Kondoh, K. Tsuda,K. Ohsawa, N. C. Kasuga, M. Oda, Bull. Chem. Soc. Jpn. 73 (2000) 1143-1152.

[57] R. Kumar, S. Obrai, A. Kaur, M. S. Hundal, H. Meehnianc, A. K. Janac, New J. Chem. 38 (2014) 1186-1198.

[58] B. T. Elie, C. Levine, I. Ubarretxena-Belandia, A. Varela-Ramírez, R. J. Aguilera, R. Ovalle, M. Contel, Eur. J. Inorg. Chem. (2009) 3421-3430.

[59] R. Uson, A. Laguna, M. Laguna, Inorg. Synth., 26 (1989) 85-91.

[60] C. Aguirre, M. C. Gimeno, A. Laguna, M. Laguna, J. M. López de Luzuriaga, F. Puente, Inorg. Chim. Acta 208 (1993) 31-36.

[61] M. Bardaji, O. Crespo, A. Laguna, A. K. Fischer, Inorg. Chim. Acta 304 (2000) 7 16. 
[62] R. Uson, A. Laguna, Inorg. Synth. 21 (1982) 71-74.

[63] SADABS, version 2.03, Bruker AXS, Inc., Madison, WI, 2000.

[64] G. M. Sheldrick, SHELXL-97, Program for Refinement of Crystal Structures, University of Göttingen, Göttingen, 1997. 
Table 1. Gram-negative bacteria. Diameter zone inhibition $(\mathrm{mm})$ in the disc diffusion test.

\begin{tabular}{|c|c|c|c|c|c|c|c|c|}
\hline \multirow{2}{*}{$\begin{array}{c}\text { Antibiotic } \\
\text { Amp }\end{array}$} & \multicolumn{4}{|c|}{ Salmonella typhimurium SV5015 } & \multicolumn{4}{|c|}{ Escherichia coli ATCC 10536} \\
\hline & \multicolumn{2}{|c|}{$100 \mu \mathrm{g} / \mathrm{ml}(0.3 \mathrm{mM})$} & \multicolumn{2}{|c|}{10} & \multicolumn{4}{|c|}{10} \\
\hline Km & \multicolumn{2}{|c|}{$50 \mu \mathrm{g} / \mathrm{ml}(0.1 \mathrm{mM})$} & \multicolumn{2}{|c|}{-} & \multicolumn{4}{|c|}{9} \\
\hline Gm & \multicolumn{2}{|c|}{$100 \mu \mathrm{g} / \mathrm{ml}(0.2 \mathrm{mM})$} & \multicolumn{2}{|c|}{11} & \multicolumn{4}{|c|}{11} \\
\hline $\mathrm{Cm}$ & \multicolumn{2}{|c|}{$30 \mu \mathrm{g} / \mathrm{ml}\left(9.2 \times 10^{-2} \mathrm{mM}\right)$} & \multicolumn{2}{|c|}{-} & \multicolumn{4}{|c|}{10} \\
\hline Complex & $25 \mathrm{mM}$ & $12.50 \mathrm{mM}$ & $6.25 \mathrm{mM}$ & $3.12 \mathrm{mM}$ & $25 \mathrm{mM}$ & $12.50 \mathrm{mM}$ & $6.25 \mathrm{mM}$ & $3.12 \mathrm{mM}$ \\
\hline 1 & - & - & - & - & Thin line & Thin line & 1 & 1 \\
\hline 3 & - & - & - & - & - & - & - & - \\
\hline \multirow[t]{2}{*}{8} & - & - & - & - & - & & & \\
\hline & $50 \mathrm{mM}$ & $25 \mathrm{mM}$ & $12.50 \mathrm{mM}$ & $6.25 \mathrm{mM}$ & $50 \mathrm{mM}$ & $25 \mathrm{mM}$ & $12.50 \mathrm{mM}$ & $6.25 \mathrm{mM}$ \\
\hline 4 & - & - & - & - & - & - & & \\
\hline 6 & - & - & - & - & - & - & & \\
\hline 9 & - & - & - & - & - & - & - & - \\
\hline 10 & - & - & - & - & - & - & - & - \\
\hline \multicolumn{9}{|l|}{11} \\
\hline 13 & 1 & 1 & 1 & 1 & 1 & 1 & 1 & 1 \\
\hline 14 & Thin line & Thin line & Thin line & Thin line & Thin line & Thin line & Thin line & Thin line \\
\hline
\end{tabular}


Table 2. Gram-positive bacteria. Diameter zone inhibition $(\mathrm{mm})$ in the disc diffusion test.

\begin{tabular}{|c|c|c|c|c|c|c|c|c|}
\hline \multirow{2}{*}{$\begin{array}{c}\text { Antibiotic } \\
\text { Amp }\end{array}$} & \multicolumn{4}{|c|}{ Listeria monocytogenes EGD-e } & \multicolumn{4}{|c|}{ Staphylococcus aureus ATCC 11632} \\
\hline & \multicolumn{2}{|c|}{$100 \mu \mathrm{g} / \mathrm{ml}(0.3 \mathrm{mM})$} & \multicolumn{2}{|c|}{19} & Amp & \multicolumn{2}{|c|}{$100 \mu \mathrm{g} / \mathrm{ml}(0.3 \mathrm{mM})$} & - \\
\hline Gm & \multicolumn{2}{|c|}{$50 \mu \mathrm{g} / \mathrm{ml}(0.1 \mathrm{mM})$} & \multicolumn{2}{|c|}{11} & Rif & \multicolumn{2}{|c|}{$0.5 \mu \mathrm{g} / \mathrm{ml}\left(6 \times 10^{-4} \mathrm{mM}\right)$} & - \\
\hline Van & \multicolumn{2}{|c|}{$10 \mu \mathrm{g} / \mathrm{ml}\left(6.9 \times 10^{-3} \mathrm{mM}\right)$} & \multicolumn{2}{|c|}{1} & Van & \multicolumn{2}{|c|}{$10 \mu \mathrm{g} / \mathrm{ml}\left(6.9 \times 10^{-3} \mathrm{mM}\right)$} & 12.5 \\
\hline Ery & \multicolumn{2}{|c|}{$10 \mu \mathrm{g} / \mathrm{ml}\left(1.3 \times 10^{-2} \mathrm{mM}\right)$} & \multicolumn{2}{|c|}{14} & DMSO & \multicolumn{2}{|c|}{ neat } & - \\
\hline Complex & $25 \mathrm{mM}$ & $12.50 \mathrm{mM}$ & $6.25 \mathrm{mM}$ & $3.12 \mathrm{mM}$ & $25 \mathrm{mM}$ & $12.50 \mathrm{mM}$ & $6.25 \mathrm{mM}$ & $3.12 \mathrm{mM}$ \\
\hline 1 & 19 & 16 & 15 & 14 & 15 & 14 & 13 & 12 \\
\hline 3 & 14 & 13 & 12 & 11 & 12 & 12.5 & 10 & 9 \\
\hline \multirow[t]{2}{*}{8} & Thin line & Thin line & Thin line & Thin line & 1 & Thin line & 1 & Thin line \\
\hline & $50 \mathrm{mM}$ & $25 \mathrm{mM}$ & $12.50 \mathrm{mM}$ & $6.25 \mathrm{mM}$ & $50 \mathrm{mM}$ & $25 \mathrm{mM}$ & $12.50 \mathrm{mM}$ & $6.25 \mathrm{mM}$ \\
\hline 4 & 8 & 8 & 9 & 9 & 11 & 11 & 11 & 11 \\
\hline 6 & 1 & 1 & 1 & 1 & 1 & 1 & 1 & 1 \\
\hline 9 & Thin line & Thin line & Thin line & Thin line & - & - & - & - \\
\hline 10 & 10 & 17 & 17 & 16 & 15 & 14 & 15 & 15 \\
\hline 11 & 19 & 11 & 11 & 12 & 14 & 14 & 14 & 14 \\
\hline 13 & 10 & 10 & 10 & 9 & - & - & - & - \\
\hline 14 & 9 & 8.5 & 8 & 1 & 10 & 9.5 & 9 & 8 \\
\hline
\end{tabular}


Table 3. MIC and MBC values (mM)

\begin{tabular}{|c|c|c|c|c|c|c|c|c|}
\hline \multirow[b]{3}{*}{ Complex } & \multirow{2}{*}{\multicolumn{2}{|c|}{$\begin{array}{c}\text { Listeria monocytogenes } \\
\text { EGD-e }\end{array}$}} & \multirow{2}{*}{\multicolumn{2}{|c|}{$\begin{array}{c}\text { Staphylococcus aureus } \\
\text { ATCC } 11632\end{array}$}} & \multirow{2}{*}{\multicolumn{2}{|c|}{$\begin{array}{c}\text { Salmonella typhimurium } \\
\text { SV5015 }\end{array}$}} & \multirow{2}{*}{\multicolumn{2}{|c|}{$\begin{array}{l}\text { Escherichia coli } \\
\text { ATCC } 10536\end{array}$}} \\
\hline & & & & & & & & \\
\hline & MIC (mM) & $\mathrm{MBC}(\mathrm{mM})$ & MIC (mM) & $\mathrm{MBC}(\mathrm{mM})$ & MIC (mM) & $\mathrm{MBC}(\mathrm{mM})$ & MIC (mM) & $\mathrm{MBC}(\mathrm{mM})$ \\
\hline 1 & 0.1 & NT & 0.1 & $>0.1$ & - & - & - & - \\
\hline 3 & - & - & - & - & - & - & - & - \\
\hline 4 & 0.2 & 0.2 & 0.2 & 5 & - & - & - & - \\
\hline 10 & 0.2 & NT & 0.2 & NT & - & - & - & - \\
\hline 11 & 0.2 & NT & 0.2 & NT & - & - & - & - \\
\hline 13 & 1 & NT & 1 & 5 & 1 & 1 & - & - \\
\hline 14 & 5 & 5 & 5 & 5 & 1 & $>1$ & - & - \\
\hline
\end{tabular}

${ }^{*} \mathrm{NT}=$ no tested 


\section{Scheme and Figure Captions}

Scheme 1. Synthesis of gold(I) and silver(I) complexes with $\mathrm{Ph}_{2} \mathrm{PNHpy}(\mathrm{L1})$. i) [AuX(tht)]; ii) $\left[\mathrm{Au}(\mathrm{OTf})\left(\mathrm{PR}_{3}\right)\right] ;$ iii) $\left[\mathrm{Au}(\mathrm{tht})_{2}\right](\mathrm{OTf}) ;$ iv) $0.5 \mathrm{Ag}(\mathrm{OTf}) ; \quad$ v) $[\mathrm{AgOTf}(\mathrm{tht})] ;$ vi) $\mathrm{Ag}(\mathrm{OTf}) ;$ vii) $\left[\mathrm{Ag}(\mathrm{OTf})\left(\mathrm{PPh}_{3}\right)_{\mathrm{n}}\right]$

Scheme 2. Synthesis of gold (I) and silver(I) complexes with [ $\left.\mathrm{Ph}_{2} \mathrm{PNH}(\mathrm{Htrz})\right]$ (L2). i) [Au(X)(tht)]; ii) $\left[\mathrm{Au}(\text { tht })_{2}\right](\mathrm{OTf})$; iii) $\left[\mathrm{Au}(\mathrm{OTf})\left(\mathrm{PR}_{3}\right)\right]\left(\mathrm{R}_{3}=\mathrm{Ph}_{3}, \mathrm{Ph}_{2}\right.$ py); iv) $\left.\mathrm{Ag}(\mathrm{OTf}) ; \mathrm{v}\right) 0.5 \mathrm{Ag}(\mathrm{OTf})$

Fig. 1. ${ }^{31} \mathrm{P}\left\{{ }^{1} \mathrm{H}\right\}$ NMR spectrum (203 K) of $\left[\mathrm{Ag}\left(\mathrm{PPh}_{3}\right)\left(\mathrm{PPh}_{2} \mathrm{NHpy}\right)\right](\mathrm{OTf})(\mathbf{8})$.

Fig. 2. Structure of the cation of complex $\left[\mathrm{Ag}\left(\mathrm{Ph}_{2} \mathrm{PNHpy}\right)_{2}\right](\mathrm{OTf})(6)$. Selected bond lengths $(\AA \AA)$ and angles for one of the independent molecules $\left({ }^{\circ}\right)$ : Ag1-N2 2.371(4) , Ag1-N2 2.371(4), Ag1P1 2.3972(14), N2-Ag1-N2 102.8(2), N2-Ag1-P1 119.41(11), N2-Ag1-P1 77.81(11), N2-Ag1-P1 77.81(11), N2-Ag1-P1 119.41(11), P1-Ag1-P1 154.07(7).

Fig. 3. Structure of the cation of complex $\left[\mathrm{Ag}\left(\mathrm{Ph}_{2} \mathrm{PNHpy}\right)\left(\mathrm{PPh}_{3}\right)_{2}\right](\mathrm{OTf})$ (9). Selected bond lengths $(\AA)$ and angles for one of the independent molecules ( $\left.{ }^{\circ}\right)$ : $\mathrm{Ag}(1)-\mathrm{N}(2)$ 2.405(5), $\mathrm{Ag}(1)-\mathrm{P}(2)$ 2.4523(16), $A g(1)-P(3)$ 2.4650(15), $A g(1)-P(1)$ 2.4948(16); N(2)-Ag(1)-P(2) 108.66(12), N(2)$A g(1)-P(3)$ 110.11(12), $P(2)-A g(1)-P(3)$ 119.42(5), N(2)-Ag(1)-P(1) 74.99(12), P(2)-Ag(1)-P(1) 121.63(6), $\mathrm{P}(3)-\mathrm{Ag}(1)-\mathrm{P}(1) 112.26(6)$.

Fig. 4. Antibiotics structures used as control drugs.

Fig. 5. Zones inhibition of complexes in disc diffusion. Plate A: S. typhimurium SV5015, plate B: E. coli ATCC 10536; plate C: S. Aureus ATCC 11632, plate D: L. monocytogenes EGD-e. First line: control antibiotics (from left to right, plate A and B: Amp, Km, Gm, Cm; plate C: Amp, Gm, Van, Ery; plate D: Amp, Rif, Van, DMSO), second line: complex 11, third line: complex 10, fourth line: complex 14. Concentrations from left to right: 50, 25, 12.5, $6.25 \mathrm{mM}$. 